\title{
A standardized educational system for radiology programs worldwide: An opinion
}

\author{
Abdulwahab Alahmari \\ Radiology Specialist, Ministry of Health, Abha, Kingdom of Saudi Arabia.
}

Corresponding Author: Abdulwahab Alahmari, Radiology Specialist, Ministry of Health, Abha, Kingdom of Saudi Arabia.

Received Date: 16 August 2021 | Accepted Date: 09 September 2021 | Published Date: 15 September 2021

Citation: A Alahmari. (2021). A standardized educational system for radiology programs worldwide: An opinion. International Journal of Clinical Case Reports and Reviews. 8(3); DOI:10.31579/2690-4861/168

Copyright: (C) 2021 Abdulwahab Alahmari, This is an open-access article distributed under the terms of the Creative Commons Attribution License, which permits unrestricted use, distribution, and reproduction in any medium, provided the original author and source are credited.

The educational system in radiology programs worldwide are different. In the American system, they offer a certificate program (Cert) then an associate degree (AAS) -in some colleges then a diploma (Dip) - after that a bachelor's degree (B.S.). A radiographer a.k.a radiologic technologist can continue to get a post-baccalaureate certificate or a master's degree (M.S.) and -rarely in America due to the shortage of Ph.D. programs- a doctorate of philosophy in radiology.

The British system in radiology programs is more advanced than the American system which offers a bachelor's degree (B.Sc.) then a post graduate certificate (PGCert) or post graduate diploma (PGDip) or a master's degree (M.Sc.) and eventually a Ph.D.

There are other countries with different educational systems in radiology which can vary from the previous two examples. All of these system does not standardized a one educational system that can work for everyone. The aim of this paper is to propose an educational system that is easy and effective.

First of all, standardize the name of all the radiology programs. Radiology is a good name which will prevent confusion with other fields. For example, radiation science can be confused with physics. Medical imaging is a broad name that include any imaging as a picture of human skin with pathology or a picture of a microscopic slide of a human specimen can be included in medical imaging. Radiologic technology or biomedical imaging is confused with IT and engineering. Radiology is a perfect name that no one will be confused with. When someone says biology no one will think it means mathematics.

Second, no degrees below bachelor degree except high school-level a.k.a a secondary education. The admission requirement is general educational diploma or high school certificate. The bachelor degree in radiology must be a 4-year long program that teaches all of the modalities (i.e. X-ray, Fluoroscopy, Computed Tomography (CT), Magnetic Resonance Imaging (MRI), Nuclear Medicine (NM), Ultrasound (US), Doppler, Catheterization lab and Angiography, etc.). This will prevent the track system which makes issues in the work market by over-saturating one track.

Third, a master's degree in radiology that offer a sub-specialty in one modality. Like a master's degree in CT alone, ultrasound alone, radiation protection alone, or Picture Archiving and Communication system (PACs) alone. The master's program can be 1 or 2 years.

Fourth, the Ph.D. program should focus on one system of the human body or a category of imaging like: $\mathrm{PhD}$ in neuroimaging, gynecology and obstetrics ultrasound, forensic imaging, pediatric imaging, or cardiovascular imaging, etc. The $\mathrm{PhD}$ program is more subspecialized program in a very small area. The $\mathrm{Ph} . \mathrm{D}$. program can be from 3 to 5 years long.

This is a very simple system and applicable which will allow more consistence in the radiology educational systems worldwide. 